\title{
EchoGéo
}

26 | 2013

Varia

\section{Enjeux et conséquences de la réglementation sur le bois-énergie au Tchad}

Ronan Mugélé

\section{(2) OpenEdition}

1 Journals

Édition électronique

URL : https://journals.openedition.org/echogeo/13620

DOI : $10.4000 /$ echogeo. 13620

ISSN : 1963-1197

Éditeur

Pôle de recherche pour l'organisation et la diffusion de l'information géographique (CNRS UMR 8586)

Référence électronique

Ronan Mugélé, "Enjeux et conséquences de la réglementation sur le bois-énergie au Tchad », EchoGéo [En ligne], 26 | 2013, mis en ligne le 19 décembre 2013, consulté le 31 juillet 2021. URL http://journals.openedition.org/echogeo/13620 ; DOI : https://doi.org/10.4000/echogeo.13620

Ce document a été généré automatiquement le 31 juillet 2021.

EchoGéo est mis à disposition selon les termes de la licence Creative Commons Attribution - Pas d'Utilisation Commerciale - Pas de Modification 4.0 International (CC BY-NC-ND) 


\title{
Enjeux et conséquences de la réglementation sur le bois-énergie au Tchad
}

\author{
Ronan Mugélé
}

1 Au Tchad, depuis 2008, il est formellement interdit d'abattre tout arbre situé sur le territoire national. Cette décision brutale décrétée par le pouvoir central s'accompagne de l'interdiction de production et de commercialisation du charbon de bois. L'objectif affiché de ces mesures est de favoriser le maintien du couvert végétal pour éloigner le spectre de la désertification. Or les ressources ligneuses représentent l'unique source d'énergie pour une écrasante majorité de la population, en forte croissance qui plus est (Sow, 1990). Comme les autres États sahéliens, le Tchad souffre en effet d'une «famine énergétique » structurelle (Magrin, 2007) : l'inaccessibilité physique ou monétaire aux sources «modernes » d'énergie (pétrole, électricité, gaz) contraint la population à privilégier le bois-énergie pour les besoins domestiques (transformation des aliments, éclairage des habitations). De fait, l'État tchadien a décidé de privilégier une approche exclusivement environnementale et extrêmement coercitive d'une pratique, la collecte du bois, qui est « symptôme du sous-développement » (Buttoud, 1995). Mais ce choix est légitimé par les opportunités politiques qu'offre la mobilisation des discours globalisés sur l'environnement dans un pays marqué entre 2004 et 2008 par d'importants troubles politico-militaires ayant gravement menacé le pouvoir du président Idriss Déby.

Inspiré des apports de la Political ecology (Gautier et Benjaminsen, 2012), cet article vise à mettre en évidence le paradoxe d'une réglementation inepte et peu applicable qui permet à l'État central de reprendre le contrôle de ses territoires ruraux mais qui ne fait que renforcer les pouvoirs locaux et les inégalités d'accès aux ressources ligneuses. Il s'appuie sur des enquêtes menées lors d'un séjour de recherche au Tchad au début de l'année 2013, combinant entretiens avec les acteurs institutionnels (administration forestière, bailleurs de fonds) et collecte de données dans deux bourgs ruraux situés à proximité du lac Tchad ${ }^{1}$. 


\section{Le bois-énergie, nouvel enjeu politique de la régulation environnementale au Tchad}

3 La règlementation sur le bois-énergie intervient à un moment particulier, marqué par le retour à la paix après plusieurs années de troubles politico-militaires. Ignorant deux des «piliers» du développement durable (aspects sociaux et économiques), ses contradictions interpellent.

\section{Une " prise de conscience » environnementale historiquement située}

4 A partir de 2004 s'ouvre une période de contestation active du pouvoir présidentiel par des groupes dissidents armés imbriqués aux rébellions du Darfour. Elle atteint son point d'orgue en avril 2006 et en février 2008 quand les rebelles parviennent dans la capitale (Magrin, 2008).

5 L'attaque rebelle du 2 février 2008 a gravement ébranlé le pouvoir présidentiel. Après avoir évité de peu sa chute, le régime s'est engagé dans des réformes considérables. Il s'est d'abord officiellement réconcilié avec le Soudan, base arrière et soutien plus ou moins discret des groupes rebelles. Revigoré par la rente pétrolière, il a ensuite considérablement renforcé son armée, signé des accords de paix avec plusieurs groupes armés et lancé une politique de grands travaux visant entre autres à développer à l'échelle nationale les infrastructures de base (routes goudronnée, hôpitaux et écoles). L'activisme de l'État s'est récemment prolongé sur la scène internationale: on peut interpréter le rôle diplomatique et militaire que s'est attribué le Tchad dans la récente crise malienne comme le prolongement extérieur de cette quête de légitimité (Magrin, 2013).

6 Dans ce contexte de retour à la paix, l'État a naturellement fait sienne la thématique environnementale, de plus en plus décisive dans les priorités des bailleurs de fonds et les grandes orientations de l'aide au développement. En 2008, la création d'une ceinture verte autour de la capitale s'accompagne de la construction du siège du Secrétariat exécutif de la Grande muraille verte ${ }^{2}$. L'organisation à Ndjamena fin 2010 de la session Afrique du $8^{\mathrm{e}}$ Forum Mondial du Développement Durable, consacrée à la sauvegarde du lac Tchad, donne une envergure inédite à la question tant débattue du devenir de ce symbole du changement climatique. Les autorités accélèrent dans le même temps l'officialisation de la création en 2010 d'une nouvelle aire protégée dans le sud-ouest du pays, le parc national de Sena Oura. Cet engagement environnemental semble consacré en 2012 par l'inscription au patrimoine de l'humanité des lacs sahariens de l'Ennedi par l'Unesco. Tous ces éléments semblent procéder d'un même mouvement de mobilisation politique autour de la thématique environnementale, qui contraste avec la période précédente au cours de laquelle le Tchad s'était montré particulièrement discret dans ce domaine (illustration 1). 
Illustration 1 - La mobilisation de discours fédérateurs sur l'environnement -ici l'avancée du désertillustre la quête de légitimité du pouvoir présidentiel au lendemain de la guerre

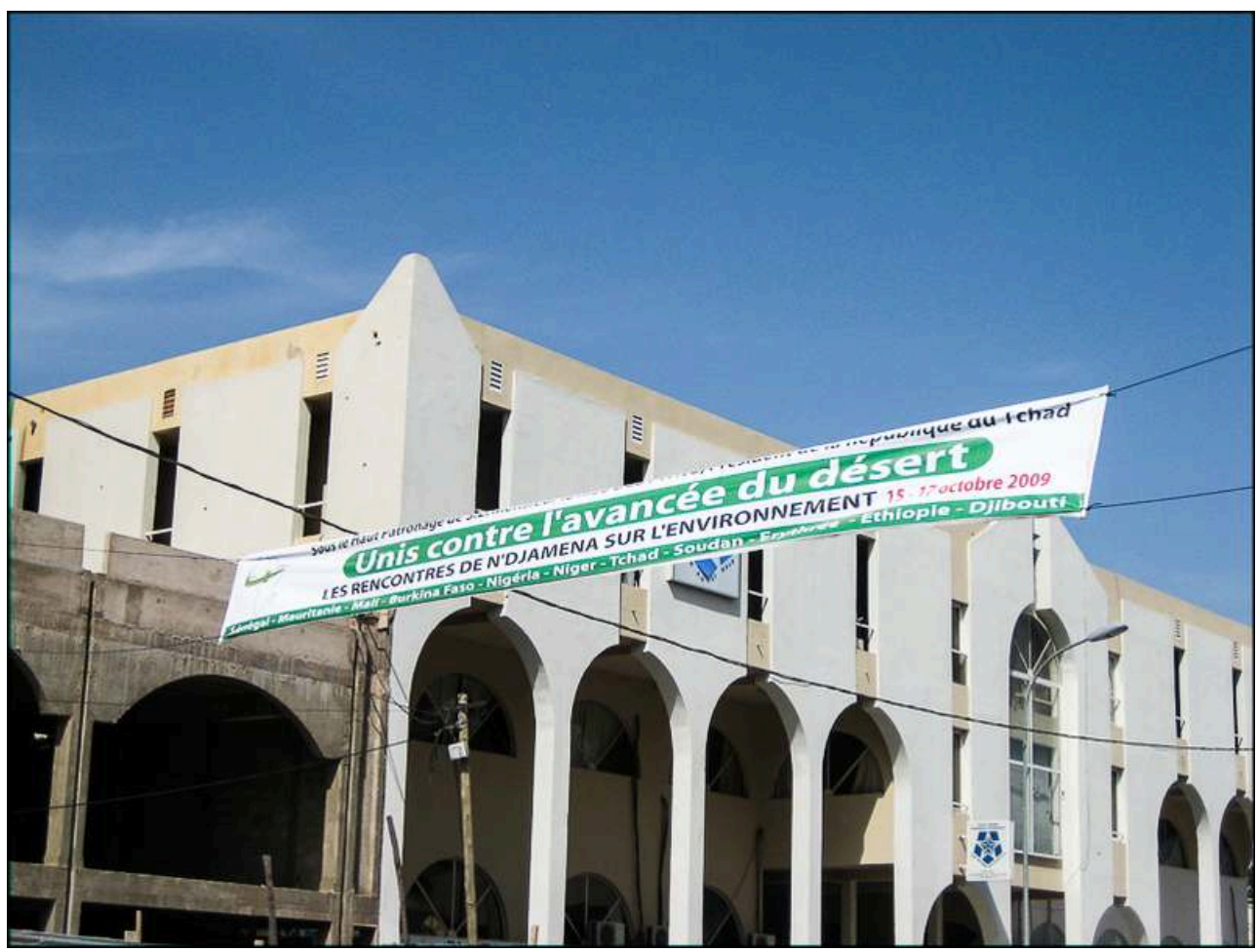

Auteur : G. Magrin, novembre 2009

En 2008, les décrets présidentiels réglementant l'exploitation des produits ligneux surgissent donc à un tournant de l'histoire récente du pays et indiquent que la sphère politique a définitivement intégré la thématique environnementale à son registre. Le discours de crise écologique au sujet de la désertification (Morel, 2006) apporte une caution pseudo-scientifique et médiatique à ces mesures radicales. L'intervention de l'État sur le bois-énergie traduit, peu après la rupture avec la Banque mondiale ${ }^{3}$, la recherche d'une respectabilité internationale par l'implication en faveur de la lutte contre le changement climatique.

\section{La réglementation sur le bois-énergie en question}

8 Dans les années 1990, le lancement du projet «Énergie domestique » sous l'égide de la FAO constitue une première initiative pour gérer durablement les ressources ligneuses en intégrant la participation des populations rurales. La création de l'Agence pour l'Énergie Domestique et l'Environnement (AEDE) en 2002, attire l'attention du gouvernement sur les conditions d'approvisionnement de Ndjamena en bois-énergie ${ }^{4}$. En s'appuyant sur les recommandations de l'AEDE, qui soulignent la pression croissante observée sur les ressources ligneuses, l'État conclut sur la nécessité de légiférer. En novembre 2004, un premier décret stipule simplement que l'usage du bois vert et du charbon de bois pour la cuisson des briques est interdit sur l'ensemble du territoire national (République du Tchad, 2004). Cette évolution reflète les mouvements de balanciers qu'opère l'État entre les approches coercitives et participatives des politiques environnementales en fonction des contingences intérieures ou extérieures, au Tchad comme ailleurs au Sahel (Haaser, 2006). 


\section{(1)}

les entreprises sur toute l'étendue du territoire national (République du Tchad, 2008a) Les « entreprises » mentionnées désignent avant tout les établissements du secteur dit informel recourant aux produits ligneux, comme les petits restaurants, les boulangeries ou les fours à briques en terre cuite. L'exportation du bois et du charbon de bois est par ailleurs déclarée illégale. Tout contrevenant s'expose à des sanctions exemplaires, allant jusqu'à 500000 FCFA. Cette mesure vise sans la nommer Ndjamena, qui connait ces dernières années une multiplication des échoppes informelles de petite restauration et un essor considérable des échanges frauduleux de bois et de charbon avec la ville frontalière camerounaise de Kousséri. Or, elle étend de jure l'interdiction à l'ensemble du territoire national : comme en 2004, l'État se substitue à la municipalité de Ndjamena et la loi, à l'arrêté municipal.

11 En décembre 2008, un décret portant mise en place d'un comité interministériel de lutte contre la dégradation de l'environnement et le braconnage entre en vigueur (République du Tchad, 2008b). Ce comité interministériel va prendre deux mesures fortes. Il décide rapidement d'interdire la coupe du bois vert ainsi que la production de charbon de bois sur l'ensemble du territoire national. De plus, il annonce la création d'une unité spéciale destinée à faire appliquer la loi sur le terrain, la Brigade nationale mobile de protection de l'environnement (BNMPE). Celle-ci est composée de militaires, gendarmes et agents des Eaux et Forêts. Dès sa création, elle se montre particulièrement active dans la lutte contre le braconnage des éléphants. Le mode d'action de cette unité paramilitaire se singularise par des méthodes expéditives. Elle a ainsi brûlé de nombreux véhicules et leur cargaison de bois en périphérie de Ndjamena ainsi que dans plusieurs petites et moyennes villes du pays (illustration 2). Véritable bras armé du Ministère de l'environnement, la BNMPE apparaît au fond comme le symbole du fonctionnement de l'État tchadien fondé sur le "gouvernement de l'entreguerres » (Debos, 2013): la multiplication des actions coup-de-poing par un corps paramilitaire éphémère -il est supprimé en 2012- vise avant tout à marquer les esprits en démontrant la puissance retrouvée de l'État. 
Illustration 2 - Les carcasses calcinées des véhicules transportant du charbon de bois sont encore visibles en périphérie de Ndjamena

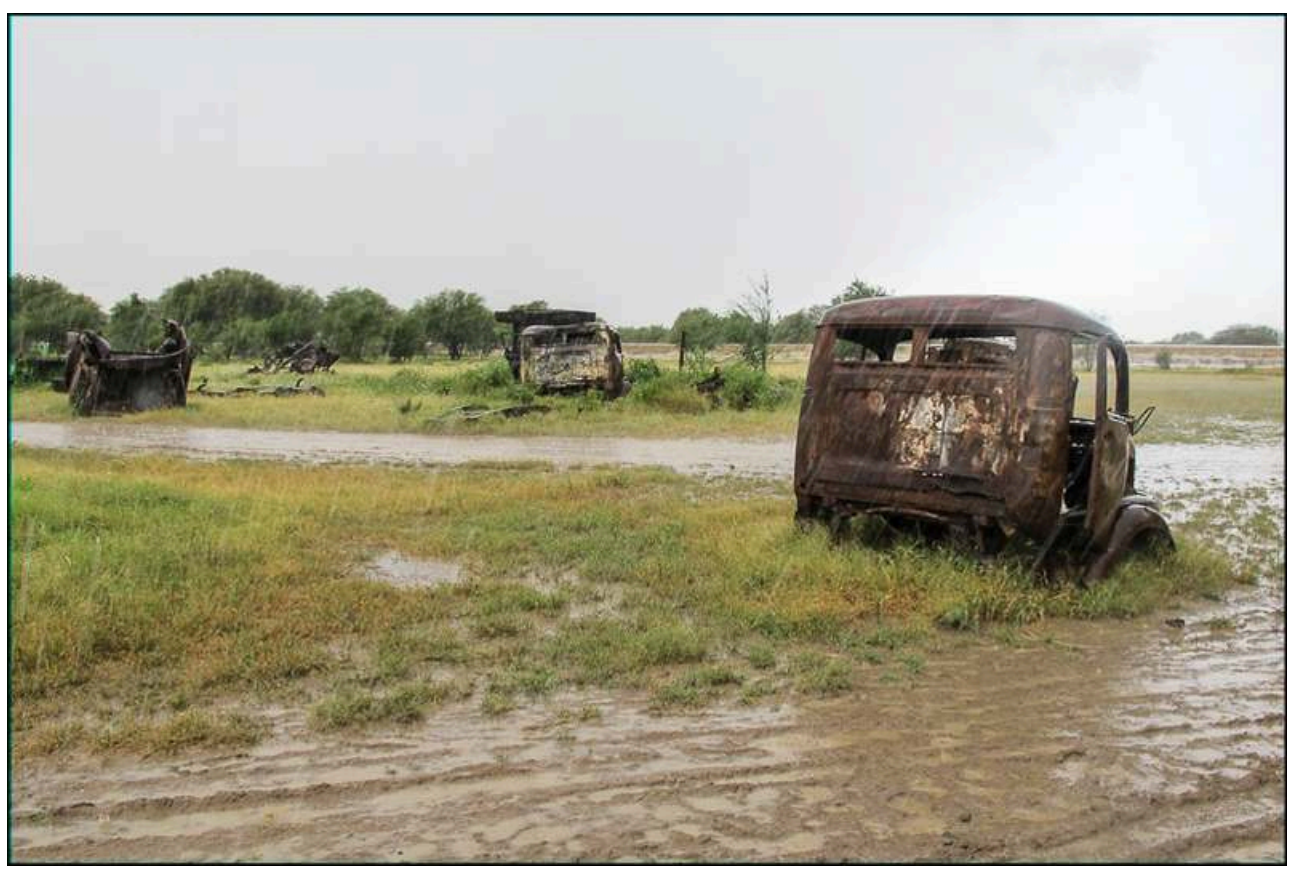

Auteur: G. Magrin, septembre 2012.

\section{Une réglementation à la légitimité douteuse}

Les motivations éminemment politiques de l'intervention de l'État au sujet du boisénergie semblent confirmées par de nombreuses incohérences sur le plan environnemental et socio-économique.

Tout d'abord, l'arbre ne se réduit pas à une réserve de combustible : au Tchad comme ailleurs en Afrique sèche, il revêt une importance majeure dans l'économie locale (Pélissier, 1980 ; Buttoud, 1995). Outre son rôle énergétique, il constitue une ressource fourragère (réserve de pâturages aériens), artisanale (bois d'œuvre) agroforestière (parcs arborés) et sociale (vertus médicinales de certaines essences). Or les interdictions reposant sur une conception restrictive des usages de l'arbre viennent entraver la gestion des ressources végétales par les populations qui en dépendent.

De plus, ces mesures vont à l'encontre du cadre législatif environnemental adopté en 2008 (République du Tchad, 2008c) et consacrant les principes du développement durable (participation de la population locale, respect de la diversité des usages des ressources naturelles, exigence de durabilité des actions gouvernementales). La promotion de la gestion intégrée des ressources naturelles semble en effet condamnée par la logique coercitive des décrets sur le bois-énergie, comme si la loi, trop abstraite et suggestive, pêchait par manque de légitimité face aux injonctions présidentielles apparues quelques mois plus tard. Cette schizophrénie de l'État, marquée par la succession de mesures s'annulant mutuellement et la multiplication des "décrets sans numéro » (Debos, 2013) est un trait caractéristique du mode de gouvernement tchadien et de son jeu avec les bailleurs de fonds.

Les mesures décrétées pâtissent surtout de l'absence d'alternative énergétique. Elles ne s'accompagnent d'aucun mécanisme de compensation, comme la subvention des 
bouteilles de gaz ou la mise en œuvre d'opérations de reboisement. Assurément peu durables ${ }^{5}$, ces mesures constituent un frein supplémentaire au développement dans un des pays où le coût de l'énergie est l'un des plus élevés au monde.

Enfin, le discours dominant sur le développement durable est paradoxalement mobilisé pour justifier de mesures coercitives héritées. Le parallèle est saisissant avec la réglementation forestière qui prévalait à l'époque coloniale, qui reposait sur les interdictions d'usages et sur l'appropriation exclusive du domaine forestier par l'administration. Plus précisément, cette réglementation reposait sur trois caractéristiques (Buttoud, 1995) : elle était spécifique, ne prenant en compte que les stricts aspects forestiers, sans considération de l'environnement économique et social ; exclusive, son objectif étant d'exclure les usagers afin de limiter la pression jugée néfaste sur les ressources forestières au nom de la défense d'un intérêt général dont seul l'État peut être le garant ; répressive, la réglementation ayant d'abord pour objectif de donner à l'autorité publique les moyens de sanctionner les contrevenants.

Les mesures prises en 2008 pour interdire les coupes de bois et le charbon apparaissent alors proprement inexplicables, voire absurdes, si l'on considère la hausse du prix des produits ligneux qu'elles ont entrainé dans la capitale ${ }^{6}$. Comment expliquer que l'État consente ainsi à se mettre à dos une écrasante majorité de la population, aussi bien rurale que citadine, dépendante au quotidien du bois-énergie?

La première hypothèse renvoie à l'instrumentalisation des choix politiques nationaux adressés au reste du monde (Blundo et de Sardan, 2007). De ce point de vue, les interdictions seraient destinées à satisfaire les conditionnalités de bailleurs de fonds de plus en plus portés sur les enjeux environnementaux ${ }^{7}$, afin de capter des flux financiers tout en améliorant l'image du pays en matière de politique environnementale. Néanmoins, l'absence de mesures compensatoires et le caractère très coercitif de la nouvelle législation viennent fortement nuancer cette interprétation. L'État tchadien n'aurait-il pas plus à gagner financièrement en transférant la gestion des ressources ligneuses aux populations rurales?

19 La seconde hypothèse concerne des enjeux de politique intérieure. D'une part, les interdictions constituent un outil de contrôle social visant à surveiller voire à rançonner les campagnes en consolidant les activités de prédation qu'offre au Tchad le statut de fonctionnaire en treillis (Debos, 2013). Cette politique visant à contrôler les ressources pour contrôler les hommes qui en dépendent, a notamment été appliquée au Mali avec l'interdiction des feux de brousse (Kull et Laris, 2009). D'autre part, les interdictions relèveraient de stratégies liées à la captation de la rente de l'État. Il s'agit de légiférer pour créer artificiellement un monopole autorisant toutes formes de corruption de la part des corps habillés de l'État et profitant à quelques personnalités influentes du régime. S'il n'existe pas de preuve tangible, les rumeurs sont nombreuses à Ndjamena au sujet de la mainmise de certains chefs militaires sur le marché du boisénergie.

\section{Le bois dans les parages méridionaux du lac Tchad: un enjeu à géométrie variable}

20 L'étude de l'exploitation du bois-énergie à l'échelle des terroirs de deux bourgs ruraux, Mani et Karal, situés dans la proche périphérie méridionale du lac Tchad, illustre les 
conditions d'application (ou d'applicabilité) des interdictions de coupe et de production de charbon.

\section{L'exploitation du bois sur les rives sud du lac Tchad}

21 L'espace regroupant le bas-Chari et les rives méridionales du lac Tchad a connu au cours des trente dernières années des mutations considérables et compte parmi les plus dynamiques du pays (Magrin et al., 2010). Cette évolution s'explique notamment par l'amélioration de l'accessibilité depuis la capitale (rôle intégrateur de la route goudronnée) et les opportunités agricoles offertes par les changements environnementaux (retrait de la nappe lacustre qui a libéré des superficies considérables en terres agricoles). Néanmoins, si les rives sud du lac Tchad apparaissent aujourd'hui comme une périphérie intégrée à Ndjamena, elles demeurent peu sensibles à la forte demande en bois-énergie émanant de la capitale, laquelle s'approvisionne bien plutôt à partir des zones boisées de son orbite méridionale (FAO, 2012). Au lac Tchad, le commerce du bois se cantonne à quelques vendeurs sur les marchés hebdomadaires, échanges insignifiants en termes de volume forestier et monétaire, ce qui permet d'observer les dynamiques locales, spécifiques et autonomes de l'exploitation des ressources ligneuses en milieu rural ${ }^{8}$.

Le cadre spatial de cette étude est centré sur Mani et Karal, deux bourgs de 5000 habitants environ et situés dans la région de Hadjer Lamis (illustration 3). Leur statut de sous-préfecture leur confère un minimum d'infrastructures publiques (lycée, dispensaire, brigade des Eaux et Forêts), mais ils abritent également le siège du sultanchef de canton dont le rôle d'autorité morale et symbolique est officiellement reconnu par les institutions publiques. Toutefois, les deux bourgs se distinguent par leurs caractéristiques socio-économiques : si Mani est un vieux site occupé par les pêcheurs Kotoko, implanté en bordure du fleuve Chari, Karal est le fief des éleveurs Arabes Choa et de nombreux cultivateurs exploitant les riches terres de décrue du lac situées quelques $10 \mathrm{~km}$ plus au nord. 
Illustration 3 -Localisation de Mani et Karal, à la charnière entre bas-Chari et rives du lac Tchad

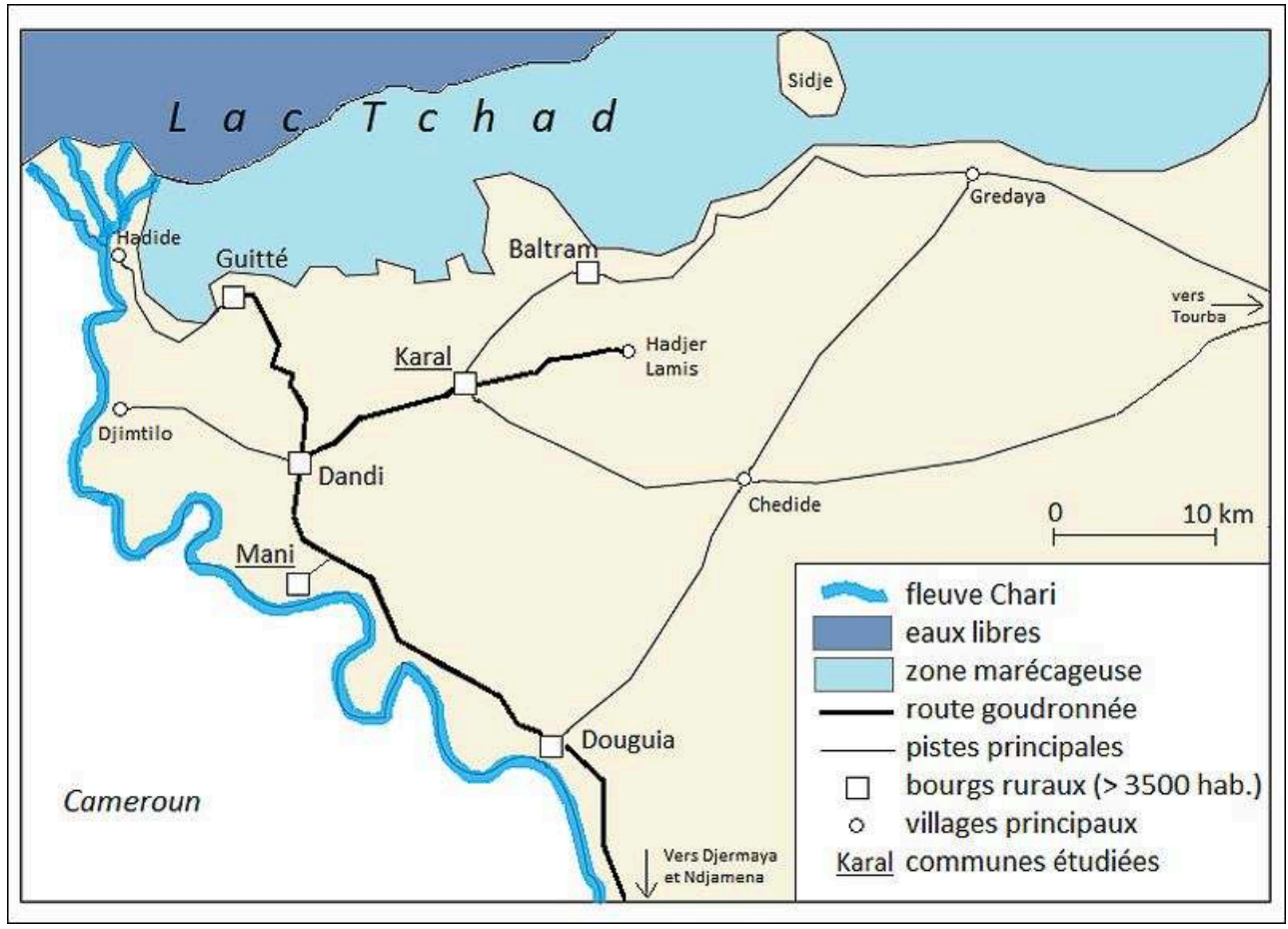

Source : R. Mugelé, 2013. dacteurs prenant part à l'exploitation du bois : les usagers regroupent les individus, souvent des femmes, qui récoltent et utilisent le bois; les autorités locales englobent pouvoirs étatiques décentralisés (préfet et sous-préfet) et chefferies coutumières (sultans et leur relais); enfin, les agents des Eaux et Forêts incarnent l'autorité légalement compétente pour faire appliquer les interdictions et sanctionner les contrevenants. L'analyse des pratiques et discours de ces trois catégories d'acteurs révèle les conséquences socio-territoriales de l'intervention de l'État dans l'exploitation du bois-énergie.

\section{Les stratégies locales de contournement de la réglementation}

Pour faire face aux nouvelles contraintes réglementaires, les habitants majoritairement démunis ont développé de multiples stratégies de contournement pour se prémunir des effets des interdictions.

La pauvreté de la population et le déficit criant d'infrastructures publiques en milieu rural interdisent le remplacement des produits ligneux par des sources énergétiques «modernes» (pétrole, électricité). Le gaz de son côté coûte cher (plus de 15000 FCFA pour une grande bouteille), n'est en vente que dans la capitale et présente des risques importants pour qui n'en maîtrise pas la technique d'utilisation. De même, rares sont 
les utilisateurs des foyers améliorés, censés limiter la vitesse de consumation des bûches et ainsi le volume de bois utilisé, mais dont le prix élevé (minimum 15000 FCFA) et les inconvénients pratiques (défauts de conception qui nuisent à l'éclairage) rendent plus attractifs les traditionnels foyers trois-briques.

Néanmoins, des combustibles de substitution sont utilisés de manière croissante, comme la noix de palmier doum (illustration 4), les déjections animales séchées et les résidus des récoltes. Ils offrent le double avantage d'être gratuits et renouvelables. Par ailleurs, les paysages portent l'empreinte de la généralisation de l'émondage des essences fourragères, pratiqué initialement par les éleveurs : partout on observe des arbres portant les stigmates de la quête de combustible (illustration 5). D'autres techniques permettent de transformer le bois vert (arbres sur pied) en bois mort, comme l'utilisation de pieux et de clous pour saigner les troncs, voire l'abattage d'arbres d'abord laissés sur place puis débités quelques semaines plus tard. Enfin, les interdictions ont forcé les usagers à étendre l'aire de prélèvement : ils vont de plus en plus loin à l'échelle de leur terroir pour trouver le bois (vert ou sec) tout en étant moins repérables par les agents forestiers. Ces pratiques transgressives sont facilitées par l'utilisation de la moto, facteur de mobilité, par le recours au téléphone portable et par la mobilisation de réseaux d'informateurs. Toutefois, aucun prélèvement de bois n'est effectué au Cameroun, de l'autre côté du fleuve, où prévalent des interdictions d'abattage similaires à celles en vigueur au Tchad, et où la végétation est plus éparse.

Illustration 4 - Les noix sèches de palmier doum (Hyphaene thebaica) sont récoltées et utilisées en combustible de substitution

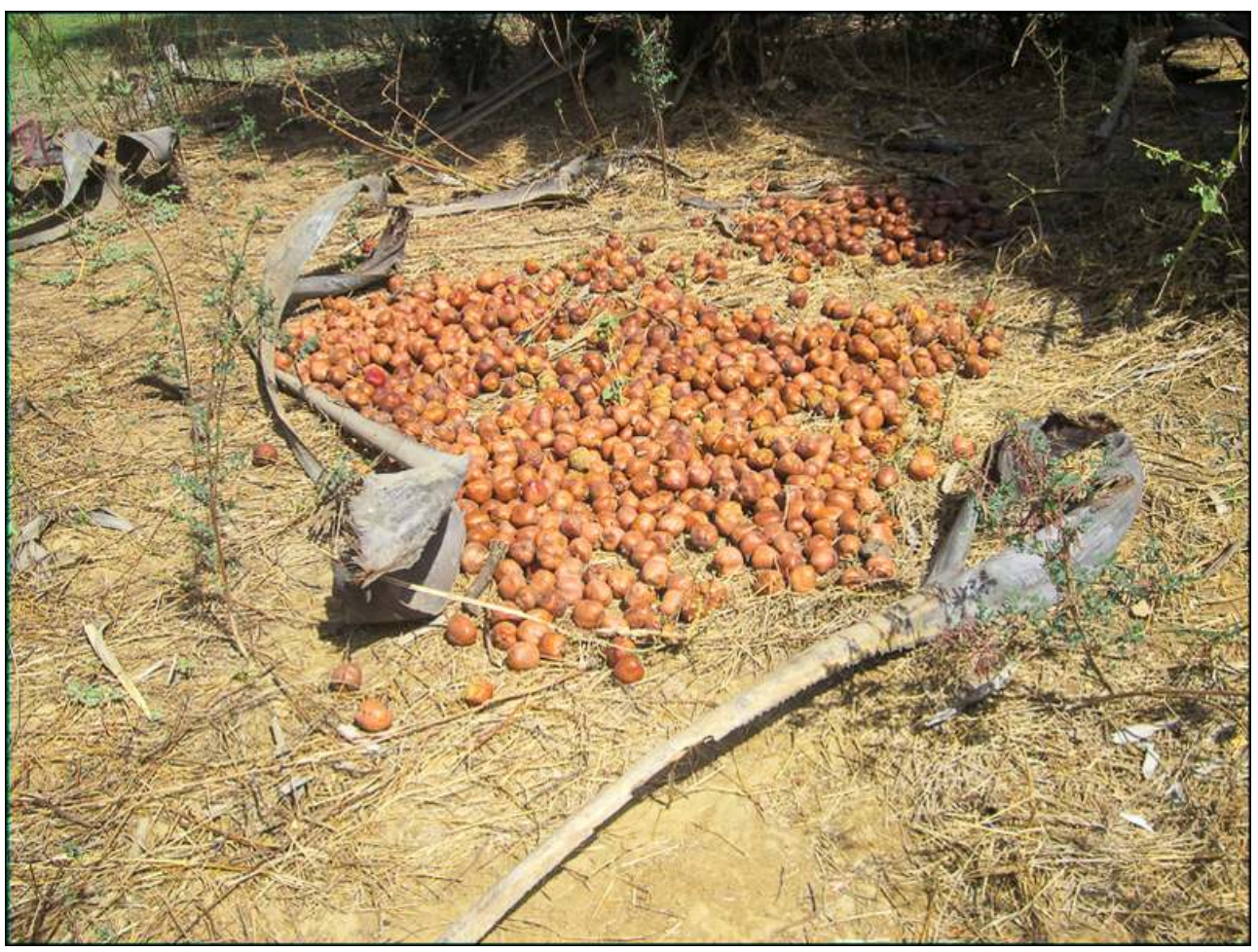

Auteur : R. Mugelé, février 2013 
Illustration 5 - L'émondage des épineux lors de la saison sèche procure quelques fagots indispensables à la cuisson des aliments

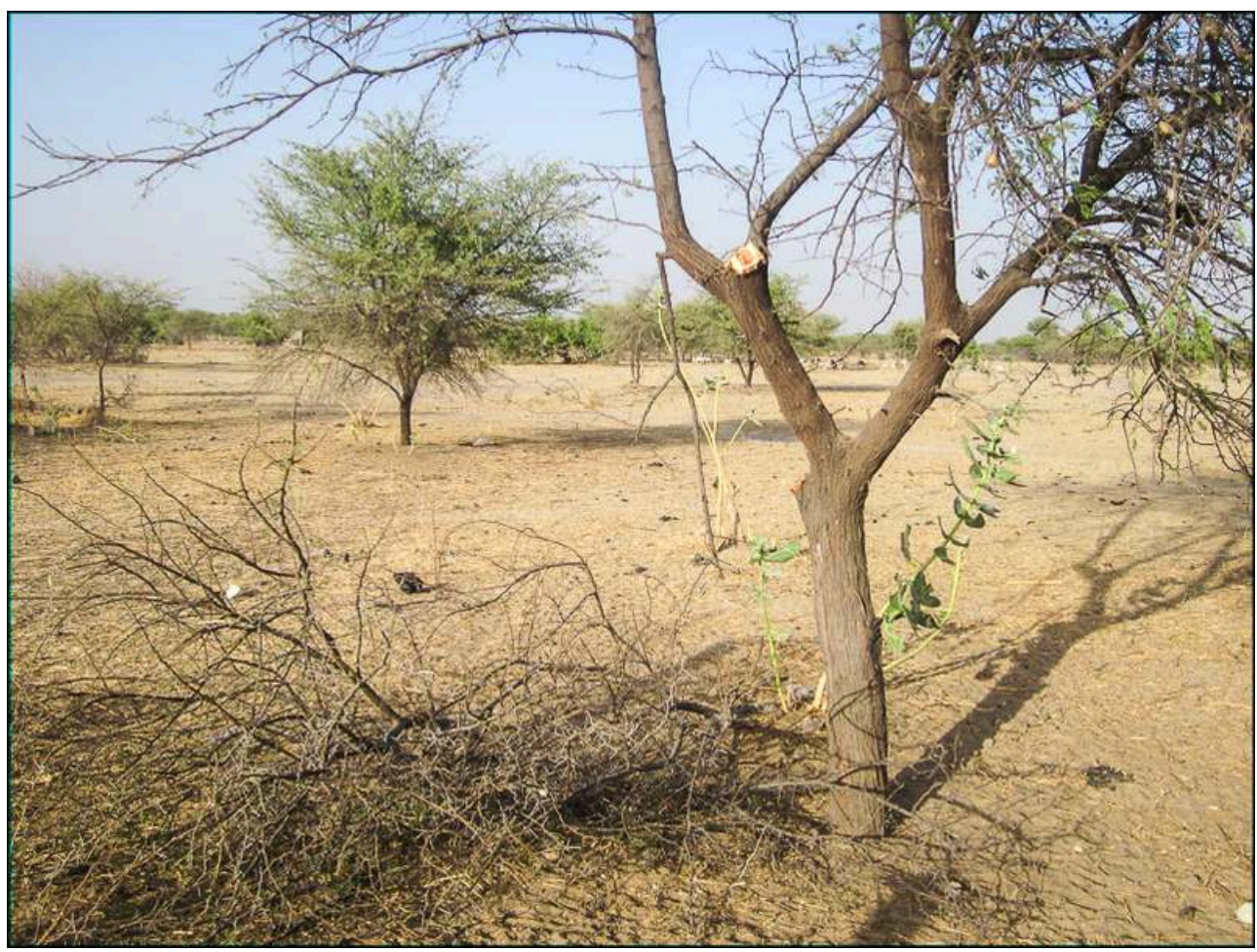

Auteur : R. Mugelé, février 2013.

\section{Opportunités et relations de pouvoir : le rôle ambigu des autorités locales}

En contribuant à faire du bois-énergie un enjeu de pouvoir à l'échelle locale, la réglementation sur le bois-énergie induit des dynamiques non maîtrisées par l'État central, comme le montre le rôle ambigu des autorités.

La décentralisation, aujourd'hui inachevée, limite grandement l'influence des souspréfets. Dans ces conditions, les initiatives d'accompagnement de la réglementation sur les produits ligneux sont inexistantes. Mais les sous-préfets, à Mani comme à Karal, n'ont pas non plus cherché à favoriser l'acceptation des mesures gouvernementales, ni même à informer la population locale de leur entrée en vigueur. Leur mutisme s'explique autant par l'impopularité pressentie de la réglementation que par la peur de perdre leur poste en dénonçant ses incohérences. Seul le préfet de Massaguet s'est prononcé lors d'une visite à Mani en 2009 contre...les feux de brousse! Officiellement, les autorités locales se font le relais des initiatives gouvernementales mais dans le même temps, elles semblent en tirer un profit personnel par l'octroi de précieuses autorisations de coupe ${ }^{9}$ voire par la production et la commercialisation clandestine de sacs de charbon de bois à destination de la capitale.

De leur côté, les autorités coutumières, dont l'autorité concurrence celle des souspréfets, adoptent une attitude ambiguë à l'égard de la réglementation. Plus particulièrement, les bulama ${ }^{10}$ paraissent tiraillés entre l'impératif de défendre les intérêts de la population et les opportunités offertes par l'adoption de la réglementation sur le bois-énergie. D'un côté, ils se présentent comme les porte- 
paroles des habitants devant le sultan. Ils sont souvent sollicités pour trouver un règlement à l'amiable avec les services forestiers pour diminuer l'amende infligée au contrevenant. Le sultan de Mani lui-même dénonce explicitement par exemple les contradictions des mesures gouvernementales, arguant de la raréfaction accélérée des ressources en bois et de la source potentielle de conflits qu'elle représente. Mais d'un autre côté, les chefs coutumiers tentent d'instrumentaliser la réglementation de la coupe du bois pour renforcer leur influence. Il est vrai que la réglementation a entraîné le départ des producteurs de charbon et restreint le commerce des produits ligneux. Or ces deux activités étaient soumises au paiement de taxes et/ou redevances, en monnaie ou en nature. L'enjeu financier et, surtout, symbolique est tel que les bulama revendiquent la création de taxes sur les produits forestiers, tout en augmentant les prélèvements sur les marchés locaux.

31 En tant que ressource naturelle à la fois banale et stratégique faisant l'objet de mesures de protection, le bois agit comme révélateur ou catalyseur des rapports de force au sein des sociétés rurales. Les interdictions créent des opportunités qui semblent renforcer les inégalités en fonction de la nature de l'exploitation du bois (besoins domestiques, cuisson des briques, défrichages) et de l'acteur concerné (paysan appauvri, commerçant incontournable, militaire intouchable). Leur application est l'objet de transactions monétaires plus ou moins importantes. Elles peuvent alimenter les rancœurs de certaines catégories de la population dénonçant les injustices et les passedroits, bien plus que la réglementation elle-même.

\section{La faible emprise territoriale de l'État et de ses relais} brigades de gendarmerie situées à bonne distance des habitations, ce qui est significatif de leur faible intégration au sein de la population locale. Celle-ci les désignent souvent comme étant des «bandits» et leur reproche pêle-mêle la fixation arbitraire du montant des amendes, le profit personnel qu'en tirent les agents et leurs complices ou encore l'application à géométrie variable des peines en fonction de l'identité du contrevenant. Il est vrai que la contradiction entre un droit répressif et une fixation de 
peines trop élevées pour que les sanctions officielles soient légitimées et donc appliquées n'est pas de nature à limiter la corruption.

Illustration 6 - Le long du fleuve Chari, la production de charbon de bois se maintient dans les zones boisées reculées ou, comme ici, dans les parcelles défrichées appartenant à de riches Ndjaménois

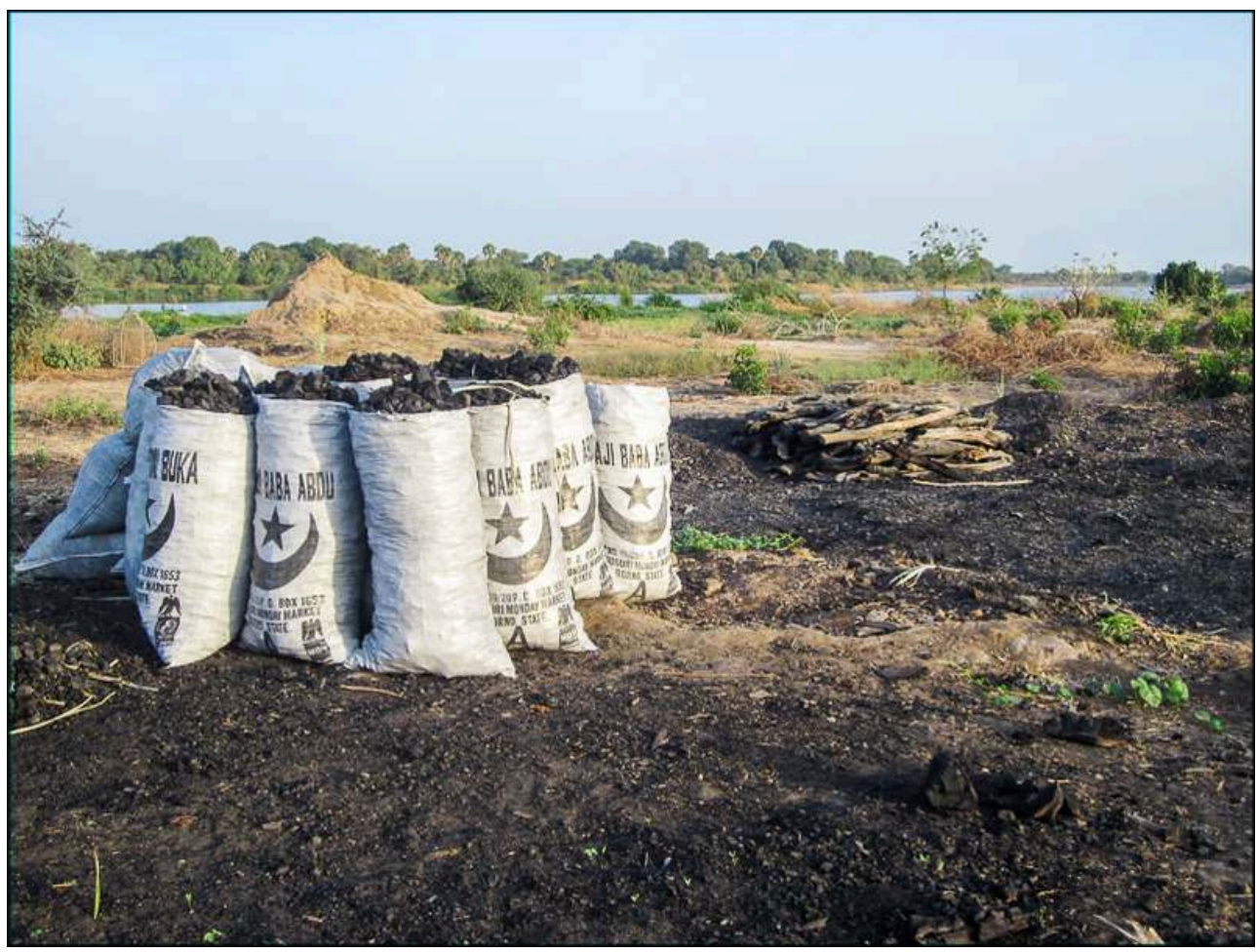

Auteur : R. Mugelé, février 2013

Ces observations amènent finalement à questionner la territorialité du contrôle effectué par les agents des services forestiers: la dimension spatiale de l'applicabilité de la réglementation. Du fait de la précarité des moyens humains et techniques dont ils disposent, les services forestiers ne sont pas en mesure de contrôler de manière homogène toute l'étendue des sous-préfectures ou des cantons. La présence de l'État est confinée de fait dans les bourgs ruraux de rang administratif, et disparaît à mesure que l'on s'en éloigne. Les axes routiers sont eux aussi peu contrôlés, sauf à l'entrée de Ndjamena où un poste de surveillance oblige les chauffeurs de poids-lourds à déclarer la nature des marchandises transportées. Le maillage territorial des services forestiers s'apparente ainsi à un réseau ou, mieux encore, à un archipel : Mani et Karal sont deux nœuds de contrôle séparés par de vastes zones échappant totalement à l'emprise de la loi. Dans ces conditions, le contrôle territorial opéré par les services forestiers, et, plus largement, par l'État tchadien contemporain, se rapproche du modèle de la «territorialité fluide » (Pourtier, 2005) et de ses déclinaisons qui prévalaient dans les empires sahéliens précoloniaux: un noyau dur, un chapelet de centres de pouvoir secondaires et des marges fluctuantes. 


\section{Conclusion}

L'année 2008 voit l'État tchadien décréter l'interdiction de l'abatage des arbres et de la production de charbon de bois sur l'ensemble du territoire national, le tout sans aucune initiative visant à en atténuer les effets. Cette décision ne peut se comprendre qu'à la lumière des conditions historiques dans lesquelles l'État a investi le champ environnemental (héritage de la coercition politique), de l'enjeu économique et symbolique que représente le bois-énergie (discours de crise environnementale au sujet de la désertification) et des contingences nationales (crise du 2 février 2008 marquant l'apogée de quatre années d'instabilité politico-militaire).

37 A l'échelle nationale, le déploiement des mesures environnementales représente un enjeu de politique intérieure lié au retour de l'État en milieu rural. Mais l'exercice du pouvoir s'avère très inégal dans l'espace : les effets de la réglementation sont les plus forts autour des centres administratifs et de quelques axes routiers. Le contraste est grand entre les injonctions d'une réglementation ambitieuse et la faiblesse structurelle de l'État tchadien. Facteur d'hétérogénéité de l'espace, la réglementation est surtout source d'inégalités sociales entre les notables locaux et les fractions les plus démunies de la population.

L'intervention de l'état dans le domaine forestier peine ainsi à masquer l'absence de politique énergétique nationale visant à pérenniser l'exploitation du bois-énergie (" produire plus plutôt que moins couper ») pour mettre la vie ou la mort de l'arbre au service du développement rural.

\section{BIBLIOGRAPHIE}

Blundo G., Olivier de Sardan J.-P., (dir.), 2007. État et corruption en Afrique. Une anthropologie comparative des relations entre fonctionnaires et usagers (Bénin, Niger, Sénégal). Paris, Apad/Karthala, $374 \mathrm{p}$.

Brami D., 2006. Le climat et l'homme, facteurs de déséquilibres des milieux sensibles sahéliens : le cas des rives sud du lac Tchad (Tchad). Thèse de doctorat de géographie, Université Paris 1 Panthéon Sorbonne, $330 \mathrm{p}$.

Buttoud G., 1995. La forêt et l'État en Afrique sèche et à Madagascar. Paris, Karthala, 247 p.

Debos M., 2013. Le métier des armes au Tchad, le gouvernement de l'entre-guerres. Paris, Karthala, $256 \mathrm{p}$.

FAO, 2012. Rapport sur l'appui à la formulation d'une stratégie et d'un plan d'action de la foresterie urbaine et périurbaine à N'Djaména. Rome, FAO, 102 p.

Gautier D., Hautdidier B., Aya I., Gazull L., 2008. Le « marché rural de bois » au Mali à l'épreuve du temps. Une innovation en friche. In Meral P., Castellanet C., Lapeyre P., (eds.), La gestion concertée des ressources naturelles : l'épreuve du temps. Paris, Gret-Karthala, p. 67-84. 
Gautier D., Benjaminsen T.A., 2012. Introduction à la political ecology. In Gautier D., Benjaminsen T. A., (dir.), Environnement, discours et pouvoir : l'approche Political ecology. Versailles, Quae, p. 5-19.

Haaser F., 2006. Synthèse sur le contrôle forestier du bois-énergie. In Bertrand A., Karsenty A., Montagne P., (eds.), Forêts tropicales et mondialisation. Paris, L'Harmattan, p. 179-193.

Kull C.A., Laris P., 2009. Fire ecology and fire politics in Mali and Madagascar. In Cochrane M.A., (dir.), Tropical fire ecology: climate change, land use and ecosystem dynamics. Berlin, Springer-Praxis, p. 171-226.

Magrin G., 2007. L’Afrique sub-saharienne face aux famines énergétiques. Echogéo, décembre 2007. [En ligne], mis en ligne le 28 février 2008, http://echogeo.revues.org/1976

Magrin G., 2008. Tchad 2008 : géographie d'une guerre ordinaire. Echogéo, mars 2008. [En ligne], Sur le Vif, mis en ligne le 13 mars 2008, http://echogeo.revues.org/2249

Magrin G., Réounodji F., Ngaressem G., Mbagogo A., Assouyouti M., 2010. Le lac Tchad et N'Ndjaména : une relation porteuse de développement ? Communication au colloque PrasacArdesac « Savanes africaines en développement. Innover pour durer ", Garoua, 20-24 avril 2009. Actes publiés sur CD Rom. http://hal.cirad.fr/cirad-00471340/fr

Magrin G., 2013. Les ressorts de l'intervention militaire tchadienne au Mali (2013). EchoGéo, juin 2013. [En ligne], Sur le Vif, mis en ligne le 28 juin 2013, http://echogeo.revues.org/13444

Morel A., 2006. Le désert avance... In Courade G., (dir.), 2006. L'Afrique des idées reçues. Paris, Belin, p. 106-111.

Pélissier P., 1980. L'arbre en Afrique tropicale : la fonction et le signe. Cahiers ORSTOM, Série Sciences Humaines, vol. XVII, $\mathrm{n}^{\circ} 3-4$.

Pourtier R, 2005. Les trois âges de la territorialité. In Antheaume B., Giraut F., (dir.), Le territoire est mort, vive les territoires! Une (re)fabrication au nom du développement. Paris, IRD, p. 40-47.

République du Tchad, décret n561/PR/PM/MEE/2004 du 15 novembre 2004 « portant interdiction de l'usage du bois vert et du charbon de bois pour la cuisson des briques ».

République du Tchad, loi n¹4/PR/2008 du 10 juin 2008 « portant régime des forêts, de la faune et des ressources halieutiques "».

République du Tchad, arrêté n 025 du 6 août 2008 " portant interdiction de l'exportation du bois et du charbon de bois et l'usage du bois et du charbon de bois dans les entreprises sur toute l'étendue du territoire national ».

République du Tchad, décret $n^{\circ} 1702 / P R / P M / 2008$ du 23 décembre 2008 « portant mise en place d'un comité interministériel de lutte contre la dégradation de l'environnement et le braconnage ».

Sow H., 1990. Le bois-énergie au Sahel. Environnement et développement. Paris, CTA-Karthala, 176p.

\section{NOTES}

1. Cet article est issu d'un mémoire de Master 2 intitulé «Quand le Tchad se met au vert... Une interprétation politique de la régulation environnementale : le cas du bois-énergie » et soutenu par l'auteur le 14 juin 2013 à l'Université Paris 1.

2. Vieux rêve panafricain, la Grande muraille verte est un projet de développement durable déployé à l'échelle de onze Etat sahéliens pour lutter contre la désertification tout en améliorant les conditions de vie des populations rurales. 
3. Cinq ans après le lancement de l'exploitation du pétrole de Doba dans le sud du pays (2003), la Banque Mondiale se retire du projet, en représailles au non-respect par le gouvernement tchadien des accords définissant les modalités de l'utilisation de la manne pétrolière.

4. Des projets semblables ont été menés dans les années 1990 au Niger et surtout au Mali avec la création des marchés ruraux de bois reposant sur une gestion décentralisée des ressources ligneuses et un système de quota d'exploitation (Gautier et al., 2008).

5. La disponibilité théorique de bois mort à l'échelle nationale pourrait satisfaire la demande du pays pour une période de 4 à 8 ans seulement (FAO, 2012).

6. À Ndjamena, où $80 \%$ des ménages continuent d'utiliser le bois comme principale source d'énergie, le prix du kilo de bois sec est passé de 35 FCFA en 2004 à 250 FCFA en 2010, et celui du charbon de bois (théoriquement importé) de 90 FCFA à 750 FCFA (FAO, 2012).

7. Plusieurs programmes de développement durable sont en cours en 2013 dans le bassin du lac Tchad, comme le Programme d'Appui au Développement Local et à la Gestion des Ressources Naturelles (Union Européenne) et le Programme de Développement Durable du Bassin du Lac Tchad (Banque Africaine de Développement).

8. Des enquêtes spécifiques sont nécessaires pour analyser l'impact de la nouvelle réglementation sur l'approvisionnement en bois-énergie de Ndjamena qui, malgré le discours de crise écologique, n'a jamais connu de véritable pénurie de combustible.

9. Officiellement, un permis est nécessaire pour défricher toute parcelle dont la superficie excède 2,5 ha.

10. Ensemble des chefs de terre entourant le sultan et servant d'intermédiaire avec la population, notamment pour la résolution des conflits.

\section{RÉSUMÉS}

Au Tchad, la préoccupation environnementale, relativement récente, prend en 2008 une ampleur nouvelle avec l'intervention de l'État en faveur de la lutte contre la désertification. L'interdiction de la coupe des arbres et de la production de charbon de bois semble répondre à un double objectif : réaffirmer l'autorité du pouvoir central au lendemain d'une profonde crise politicomilitaire et capter de nouvelles rentes environnementales mondialisées. Le cas des rives méridionales du lac Tchad révèle les transformations locales des rapports de pouvoir et des pratiques liées à l'exploitation du bois-énergie. L'emprise inégale du contrôle territorial exercé par les services forestiers en limite toutefois la portée.

In 2008, in Chad, authorities implemented a new set of environmental regulation over the use and exploitation of woodfuels. Cutting wood and producing charcoal were declared illegal. This radical measure seems to obey to an ambivalent logic. On the one hand, it aims at fighting against desertification. On the other hand, it is used to enforce the State's authority on the aftermath of major political troubles. The case-study of southern shores of Lake Chad reveals transformations in the dynamics of local power and resource exploitation, even though many places remain beyond the capacity of authorities to control. 
INDEX

Mots-clés : Bois-énergie, politique environnementale, désertification, political ecology, Tchad

Keywords : Woodfuels, environmental policies, desertification, political ecology, Chad

\section{AUTEUR}

\section{RONAN MUGÉLÉ}

Ronan Mugelé, ronan.mugele@gmail.com, est doctorant en géographie à l'Université Paris 1 Panthéon Sorbonne et rattaché à l'UMR 8586 Prodig. 